\title{
Responses of IgE, IgG1, and IgG4 to concanavalin A-binding Blomia tropicalis antigens in allergic patients
}

K.C. Almeida ${ }^{1}$, D.A.O. Silva ${ }^{1}$, M.L. Gennari-Cardoso ${ }^{1}$, J.P. Cunha-Júnior², R. Alves ${ }^{1}$, L.H. Ynoue ${ }^{1}$, R.O. Resende ${ }^{1}$, S.J. Sung 3 and E.A. Taketomi ${ }^{1}$

\author{
${ }^{1}$ Laboratório de Alergia e Imunologia Clínica, Instituto de Ciências Biomédicas, \\ Universidade Federal de Uberlândia, Uberlândia, MG, Brasil \\ 2Divisão de Imunologia e Microbiologia, Departamento de Ciências Biológicas, \\ Universidade Estadual de Santa Cruz, Ilhéus, BA, Brasil \\ ${ }^{3}$ Division of Rheumatology and Immunology, Health Sciences Center, \\ University of Virginia, Charlottesville, VA, USA
}

Correspondence

E.A. Taketomi

Disciplina de Imunologia

Instituto de Ciências Biomédicas

Universidade Federal de Uberlândia

Av. Pará, 1720

38400-902 Uberlândia, MG

Brasil

Fax: +55-34-3218-2333

E-mail: taketomi@ufu.br

Research supported by CAPES, CNPq and FAPEMIG.

Received January 30, 2006

Accepted July 21, 2006

\begin{abstract}
Blomia tropicalis $(\mathrm{Bt})$ and Dermatophagoides pteronyssinus $(\mathrm{Dp})$ are the prevalent house dust mites in tropical countries and are associated with allergic diseases. Glycosylated antigens are highly immunogenic and involved in different pathologies. We evaluated the presence of $\mathrm{IgE}, \mathrm{IgG1}$, and IgG4 to concanavalin A-binding antigens (Bt-Con-A) isolated from Bt-total extract in sera of allergic and non-allergic subjects. Bt-total and Bt-Con-A extracts were evaluated by SDSPAGE and ELISA for reacting with IgE, IgG1, and IgG4 in sera of 121 patients with allergic rhinitis and 36 non-allergic individuals. All subjects were skin prick tested with Bt-total extract and inhibition tests were performed for $\mathrm{IgE}, \mathrm{IgG} 1$, and $\mathrm{IgG} 4$ using both extracts (Bttotal and Bt-Con-A). Skin prick test showed that $58 \%$ of the patients were sensitized to $\mathrm{Bt}(\mathrm{Bt}+)$, with $52 \%$ reactive to both mites (Bt and $\mathrm{Dp})$ and $6 \%$ to Bt only. A broad spectrum of proteins (14-152 kDa) was visualized in Bt-total and components $>27 \mathrm{kDa}$ for the Bt-Con-A extract. ELISA showed a similar profile of IgE, IgG1 and IgG4 levels in response to Bt-total and Bt-Con-A extracts in different groups, although $\mathrm{Bt}+$ patients showed a lower IgG4 reactivity to Bt-Con-A extract. Specific $\operatorname{IgG1}$ levels were higher in $\mathrm{Bt}+$ patients than in control subjects, and IgG4 levels showed no significant difference among groups. ELISA inhibition showed a partial IgE and total IgG1 and IgG4 cross-reactivity with Dp extract for Bt-total and Bt-Con-A extracts. We conclude that Con-A-binding components isolated from Bt constitute major allergens and are involved in both allergen sensitization (IgE response) and homeostasis maintenance (IgG1 and IgG4 responses).
\end{abstract}

Key words

- Allergy

- Blomia tropicalis

- Concanavalin A

- Immunoglobulin E

- Immunoglobulin G1

- Immunoglobulin G4

\section{Introduction}

Blomia tropicalis $(\mathrm{Bt})$ and Dermatophagoides pteronyssinus (Dp) are the most prevalent domestic mites in house dust in tropical and subtropical climates $(1,2)$. The role of house dust mites (HDMs) as a source of allergens and their contribution to allergic diseases, particularly asthma and rhinitis, have been recognized for many years (3). 
Sensitization to indoor allergens is the most important independent risk factor associated with asthma. Positive skin tests and/or serum IgE antibodies to HDM have been widely demonstrated in genetically predisposed individuals (4).

The role of IgG antibodies to HDM allergens in the pathogenesis of allergic diseases is not clear. Several investigators have attributed a protective activity to IgG subclasses, mainly $\operatorname{IgG} 4$, which is produced as a result of a prolonged natural antigenic exposure and could neutralize the antigen or block the antibody, thus supporting the immunotherapy $(5,6)$. Smith et al. (7) have investigated the IgG and IgG1-4 antibody responses to Der p 2 in children from high and low exposure groups by ELISA, demonstrating that $\geq 90 \%$ of the children had Der p 2specific IgG antibodies, although the levels of both total specific IgG and IgG1-4 antibodies were substantially higher in the high exposure group. Recently, we (8) demonstrated IgE, IgG1 and IgG4 antibody responses to $B$. tropicalis in patients with respiratory allergy, emphasizing that our patients seem to be more frequently sensitized to $B$. tropicalis high-molecular weight (54$104 \mathrm{kDa}$ ) components than to the low-molecular weight (11-15 kDa) allergens, which have been detected predominantly in other studies $(9,10)$.

Glycosylated antigens have been shown to play a crucial role in different pathologies and to be highly immunogenic (11-13). Mannanrich glycosylated components derived from Candida albicans have been recognized by IgE antibodies in patients with allergic manifestations including asthma, rhinitis, and atopic dermatitis (14). In allergic diseases, previous studies have demonstrated that tridacnin- but not concanavalin A (Con-A)-binding highmolecular weight allergens isolated from $D$. farinae reacted strongly with sera from mitesensitized subjects (15).

The purpose of the present study was to isolate glycosylated components from a to- tal extract of $B$. tropicalis using a Con-A affinity column. We evaluated the responses of $\mathrm{IgE}, \mathrm{IgG} 1$, and $\mathrm{IgG} 4$ antibodies to these Con-A reactive components from $B$. tropicalis in mite-sensitized patients. We also evaluated the cross-reactivity of these antibodies to total extracts of $\mathrm{Dp}$.

\section{Material and Methods}

\section{Subjects and skin prick test}

A total of 121 adult patients with allergic rhinitis (AR) with/without intermittent or persistent mild-to-moderate asthma were selected on the basis of the history of respiratory symptoms related to house dust exposure, physical examination, and spirometry when indicated $(16,17)$ at the Allergy and Clinical Immunology Unit, Federal University of Uberlândia, Uberlândia, MG, Brazil. Patients underwent skin prick testing (SPT) with Bt and Dp extracts. A mean wheal diameter more than $3 \mathrm{~mm}$ larger than the negative control (diluent) was considered to be positive. Thirty-six healthy adult subjects were included as a control group. In parallel, blood samples were collected from all subjects for serological testing. The study was approved by the Ethics Committee in $\mathrm{Hu}$ man Research of the Federal University of Uberlândia and written informed consent was obtained from all individuals.

\section{Mite extracts}

Total extracts of $\mathrm{Bt}$ and $\mathrm{Dp}$ were obtained from mite bodies provided by Dr. Federico Montealegre, Ponce School of Medicine, Porto Rico, USA, as described elsewhere (8). Briefly, $5 \mathrm{~g}$ dried mite powder was triturated in liquid nitrogen to disrupt the mites and allergens were extracted overnight at $4^{\circ} \mathrm{C}$ in $50 \mathrm{~mL} 5 \mathrm{mM}$ borate-buffered saline, $\mathrm{pH}$ 8.0, containing protease inhibitors $(50 \mu \mathrm{g} / \mathrm{mL}$ leupeptin, $1.6 \mathrm{mM}$ phenylmethylsulfonyl fluoride, $1 \mathrm{mM}$ benzamidine, 
and $10 \mu \mathrm{g} / \mathrm{mL}$ aprotinin). After centrifugation $\left(30,000 \mathrm{~g}\right.$ for $45 \mathrm{~min}$ at $\left.4^{\circ} \mathrm{C}\right)$, the supernatant was dialyzed (Amicon, Ym-10, W.R. Grace \& Co., Beverly, MA, USA) against phosphate-buffered saline (PBS) and the protein concentration was determined by the method of Lowry et al. (18). The Bt and Dp total extracts contained 9 and $4.5 \mathrm{mg}$ protein/mL, respectively.

Isolation of Con-A-reactive components from total extracts of $B$. tropicalis

Glycosylated protein from total extracts of $\mathrm{Bt}$ (18 $\mathrm{mg}$ total protein) were isolated by affinity chromatography using $5 \mathrm{~mL}$ Con-A Sepharose (Amersham Pharmacia Biotech AB, Uppsala, Sweden) previously equilibrated with PBS. Con-A reactive components were eluted with $50 \mathrm{mM} \alpha$-D-methylmannopyranoside (Ferro Pfanstiehl Laboratories Inc., Waukegan, IL, USA) and column effluents were monitored at $280 \mathrm{~nm}$. Con-A-unbound (Bt-void; eluted in void volume) and Con-A-bound (Bt-Con-A) fractions were concentrated and dialyzed against PBS (Amicon, Ym-10, W.R. Grace \& Co.). The protein content was determined by the method of Lowry et al. (18) and corresponded to $490 \mu \mathrm{g} / \mathrm{mL}$ for Bt-Con-A and 3,800 $\mu \mathrm{g} /$ $\mathrm{mL}$ for Bt-void.

\section{SDS-PAGE of B. tropicalis extracts}

B. tropicalis whole extract (Bt-total) and extracts obtained after fractionating by affinity chromatography (Bt-void and Bt-ConA) were electrophoresed on $12 \%$ sodium dodecylsulfate polyacrylamide gel (SDSPAGE) using $20 \mu$ g extract per lane and a mini-gel system (Mighty Small Multiple Caster, Hoefer Pharmacia Biotech Inc., San Francisco, CA, USA), under non-reducing conditions, according to the method of Laemmli (19). The gel was subsequently stained with Coomassie brilliant blue (Sigma, St. Louis, MO, USA).

\section{Measurement of IgE, IgG1 and IgG4 by ELISA}

IgE-, IgG1-, and IgG4-ELISA were performed as described elsewhere (8). Briefly, microtiter plates were coated with $1 \mu \mathrm{g} / \mathrm{well}$ Bt-total or Bt-Con-A extracts, blocked with PBS containing $0.05 \%$ Tween 20 and $1 \%$ bovine serum albumin, and then incubated with serum samples diluted 1:2 (IgE) or 1:5 (IgG1 and IgG4). Biotinylated anti-human IgE (1:500; Kierkegaard \& Perry Lab., Gaithersburg, MD, USA), anti-human IgG1 (1:250; Sigma) or anti-human IgG4 (1:1000; Sigma) were added, followed by streptavidin-peroxidase (1:500; Sigma). The assay was developed by adding $10 \mathrm{mM}$ 2,2'-azinobis-3-ethyl-benzthiazoline sulfonic acid (Sigma) and $0.03 \% \mathrm{H}_{2} \mathrm{O}_{2}$. Absorbance (Abs) was determined in a plate reader (Titertek Multiskan, Flow Laboratories, McLean, VA, USA) at $405 \mathrm{~nm}$ and antibody titers were arbitrarily expressed as ELISA index (EI) according to the formula: $\mathrm{EI}=$ sample Abs/ cut-off, where cut-off was established as mean Abs of 3 negative control sera plus 3 standard deviations. EI values $>1.2$ were considered positive for excluding borderline reactivity values close to $\mathrm{EI}=1.0$.

\section{Competitive inhibition ELISA}

To evaluate the degree of cross-reactivity between $\mathrm{Bt}$ and $\mathrm{Dp}$ extracts, competitive inhibition assays were performed as previously described (8). Briefly, Bt-total, BtCon-A and Dp-total extracts were serially diluted 10 -fold $\left(10^{2}\right.$ to $\left.10^{-4} \mu \mathrm{g} / \mathrm{mL}\right)$ and preincubated overnight at $4^{\circ} \mathrm{C}$ with six pooled sera (diluted 1:5) obtained from patients with high levels of specific antibodies to $B$. tropicalis. Con-A was also included as negative control.

The inhibitor-serum mixtures were added to microtiter plates previously coated with Bttotal or Bt-Con-A and assayed by ELISA for $\mathrm{IgE}, \mathrm{IgG} 1$, and $\mathrm{IgG} 4$ as described above. Sera incubated with diluent only were used as posi- 
tive controls and results are reported as percent inhibition and calculated as follows: [1.0 - (sample Abs/positive control Abs)] x 100.

\section{Statistical analysis}

Statistical analysis consisted of determinations of means for the levels of antibodies to $B$. tropicalis and the differences between the means were analyzed by the KruskalWallis test using the Dunn multiple comparison test as post hoc test. Positivity rates of antibodies to B. tropicalis were compared using the Fisher exact test. Correlation between the levels of antibodies to B. tropicalis was determined in sera of patients with positive SPT $(\mathrm{Bt}+)$ using the Spearman correlation test. Differences were considered statistically significant when $\mathrm{P}<0.05$.

\section{Results}

\section{SPT to B. tropicalis and D. pteronyssinus}

A total of 157 subjects (121 AR patients and 36 healthy individuals) were skin prick tested. All healthy individuals (control group) showed a negative SPT to HDM extracts. SPT results for B. tropicalis and Dp in 121 AR patients are summarized in Table 1. Seventy (58\%) patients had positive SPT to

Table 1. Results of the skin prick test (SPT) in response to Blomia tropicalis (Bt) and/or Dermatophagoides pteronyssinus (Dp) extracts in 121 patients presenting allergic rhinitis.

SPT results

$\mathrm{Bt}+$ $\mathrm{Bt}+/ \mathrm{Dp}+$ $70(57.9 \%)$ $\mathrm{Bt}+/ \mathrm{Dp}-$ $63(52.1 \%)$

$\mathrm{Bt}-$ $\mathrm{Bt}-/ \mathrm{Dp}+$ $\mathrm{Bt}-/ \mathrm{Dp}-$

$51(42.1 \%)$ $19(15.7 \%)$ $32(26.4 \%)$

Data are reported as the number of patients with percent in parentheses. $+=$ positive; $-=$ negative
B. tropicalis, 63 (52\%) were positive to both mites ( $\mathrm{Bt}+/ \mathrm{Dp}+)$, and $7(5.8 \%)$ were positive to $B$. tropicalis only ( $\mathrm{Bt}+/ \mathrm{Dp}-)$. In contrast, of 51 (42\%) patients with negative SPT to $B$. tropicalis, 19 (16\%) were reactive to $\mathrm{Dp}$ only (Bt-/Dp+) and $32(26 \%)$ showed no reactivity to either mite (Bt-/Dp-).

\section{Con-A-reactive components from total B. tropicalis extracts}

When Bt-total extract was fractionated on a Con-A-Sepharose affinity column the chromatographic profile showed a Con-Aunbound fraction (Bt-void) and two peaks after eluting with $50 \mathrm{mM}$ methyl $\alpha$-D-mannopyranoside consisting of Con-A-bound fractions (Bt-Con-A; Figure 1A). When analyzed by SDS-PAGE and stained with Coomassie brilliant blue (Figure 1B), Bt-total extract showed a broad spectrum of protein components with relative molecular masses ranging from 14 to $152 \mathrm{kDa}(14,22,24,29$, $37,50,66,93,123$, and $152 \mathrm{kDa})$. The Btvoid fraction showed protein components below $30 \mathrm{kDa}(14,22,24$, and $29 \mathrm{kDa})$ while bands above $27 \mathrm{kDa}(27,29,37,50,66,93$, 123 , and $152 \mathrm{kDa}$ ) were visualized in the BtCon-A fraction.

\section{IgE, IgG1, and IgG4 antibodies to B. tropicalis}

Levels of $\operatorname{IgE}, \operatorname{IgG} 1$ and $\mathrm{IgG} 4$ antibodies to Bt-total extract and Bt-Con-A fraction determined by ELISA in serum from patients with positive $(\mathrm{Bt}+)$ and negative $(\mathrm{Bt}-)$ SPT to B. tropicalis and from control subjects are illustrated in Figure 2.

Levels of specific IgE antibody to Bt-total (Figure 2A; ELISA index) were significantly higher in $\mathrm{Bt}+(\mathrm{EI}=2.32)$ than in $\mathrm{Bt}-(\mathrm{EI}=0.82)$ patients or control $(\mathrm{EI}=0.78)$ individuals $(\mathrm{P}<$ 0.05). Similarly, levels of IgE antibodies to BtCon-A were significantly higher in $\mathrm{Bt}+(\mathrm{EI}=$ 1.81) than in Bt- $(\mathrm{EI}=0.84)$ patients or controls $(\mathrm{EI}=0.77)(\mathrm{P}<0.05)$. 
Sera from Bt+ patients had significantly higher levels of IgG1 antibodies to Bt-total $(\mathrm{EI}=4.56)$ than control sera $(\mathrm{EI}=3.14)(\mathrm{P}<$ $0.05)$, while Bt- patients $(\mathrm{EI}=3.75)$ did not present any significant difference from the $\mathrm{Bt}+$ and control groups $(\mathrm{P}>0.05$; Figure 2B). The same was observed for Bt-Con-A, with significantly higher levels of IgG1 in $\mathrm{Bt}+$ patients $(\mathrm{EI}=4.54)$ than in control subjects $(\mathrm{EI}=3.01 ; \mathrm{P}<0.05)$, without a significant difference between the Bt- $(\mathrm{EI}=3.74)$ and $\mathrm{Bt}+$ patients or the control group $(\mathrm{P}>$ 0.05).

As demonstrated in Figure 2C, only Bt+ patients showed significantly higher levels of IgG4 antibodies to Bt-total $(\mathrm{EI}=2.13)$ in comparison to Bt-Con-A (EI = 1.23; $\mathrm{P}<$ 0.05 ), with no significant difference between $\mathrm{Bt}+$ and Bt- patients and control subjects for either extract.

Sera assayed on microtiter plates previously coated with Con-A (negative control for the Bt-Con-A extract) did not show any reactivity, with EI values below 1.2 (data not shown).

\section{Positivity of IgE, IgG1, and IgG4 to $B$. tropicalis}

The percentages of positive $\operatorname{IgE}, \operatorname{IgG} 1$ and IgG4 antibodies to Bt-total and Bt-Con-

A extracts determined by ELISA in patients with positive $(\mathrm{Bt}+)$ and negative (Bt-) SPT to B. tropicalis and control subjects are shown in Figure 3.

For Bt-total extract (Figure 3A), all patient groups showed significantly higher $\mathrm{IgG} 1$ positivity than $\mathrm{IgE}$ and $\mathrm{IgG} 4$ positivity $(\mathrm{P}<$ 0.05). In $\mathrm{Bt}+$ patients, positivity for $\mathrm{IgE}$ and IgG4 was identical, while in Bt- patients and control subjects, IgG4 positivity was significantly higher than for $\operatorname{IgE}(\mathrm{P}<0.05)$. The same was observed for the Bt-Con-A extract (Figure 3B), except for Bt+ patients who showed significantly higher positivity for $\operatorname{IgE}(47 \%)$ than for IgG4 (31\%; P < 0.05). When comparing the antibody positivity rates in each group between the Bt-total and BtCon-A extracts, only the $\mathrm{Bt}+$ group showed a significant decrease in percentage of positive IgG4 to Bt-Con-A (60 to 31\%; $\mathrm{P}<0.05$ ).

\section{Correlation and association between IgE, IgG1, and IgG4 antibodies with Bt-total and Bt-Con-A}

To determine the reactivity of antibodies to Bt-total extract as compared to the ConA-reactive components (Bt-Con-A), we investigated the correlation between the levels of $\mathrm{IgE}, \mathrm{IgG} 1$ and $\mathrm{IgG} 4$ antibodies to both extracts in sera of patients with positive SPT

A

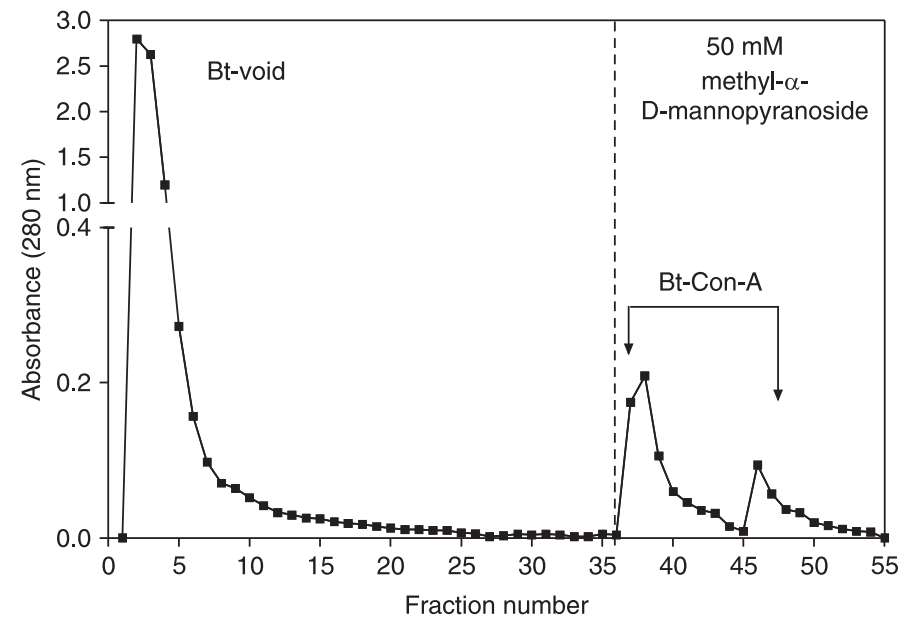

B

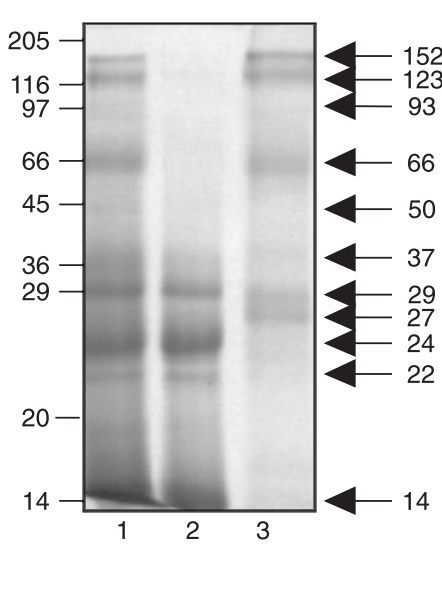

Figure 1. $A$, Isolation of concanavalin $A$ (Con-A)-reactive proteins from Blomia tropicalis whole extract (Bt-total) by affinity chromatography on ConA-Sepharose. Bt-total extract (18 mg) was applied to a $5 \mathrm{~mL}$ Con-A-Sepharose column. The column was washed with PBS and then eluted (vertical dashed line) with $50 \mathrm{mM}$ methyl- $\alpha$-Dmannopyranoside. Effluent protein was detected by absorbance at $280 \mathrm{~nm}$. B, Electrophoretic profile (12\% SDS-PAGE) of $B$. tropicalis extracts stained with Coomassie blue. Lane 1, Bt-total; lane 2, Bt-void; lane 3, Bt-Con-A. Electrophoretic mobility of molecular mass $(\mathrm{kDa})$ standards are indicated on the left of panel $B$ and $B$. tropicalis protein components are indicated on the right. 

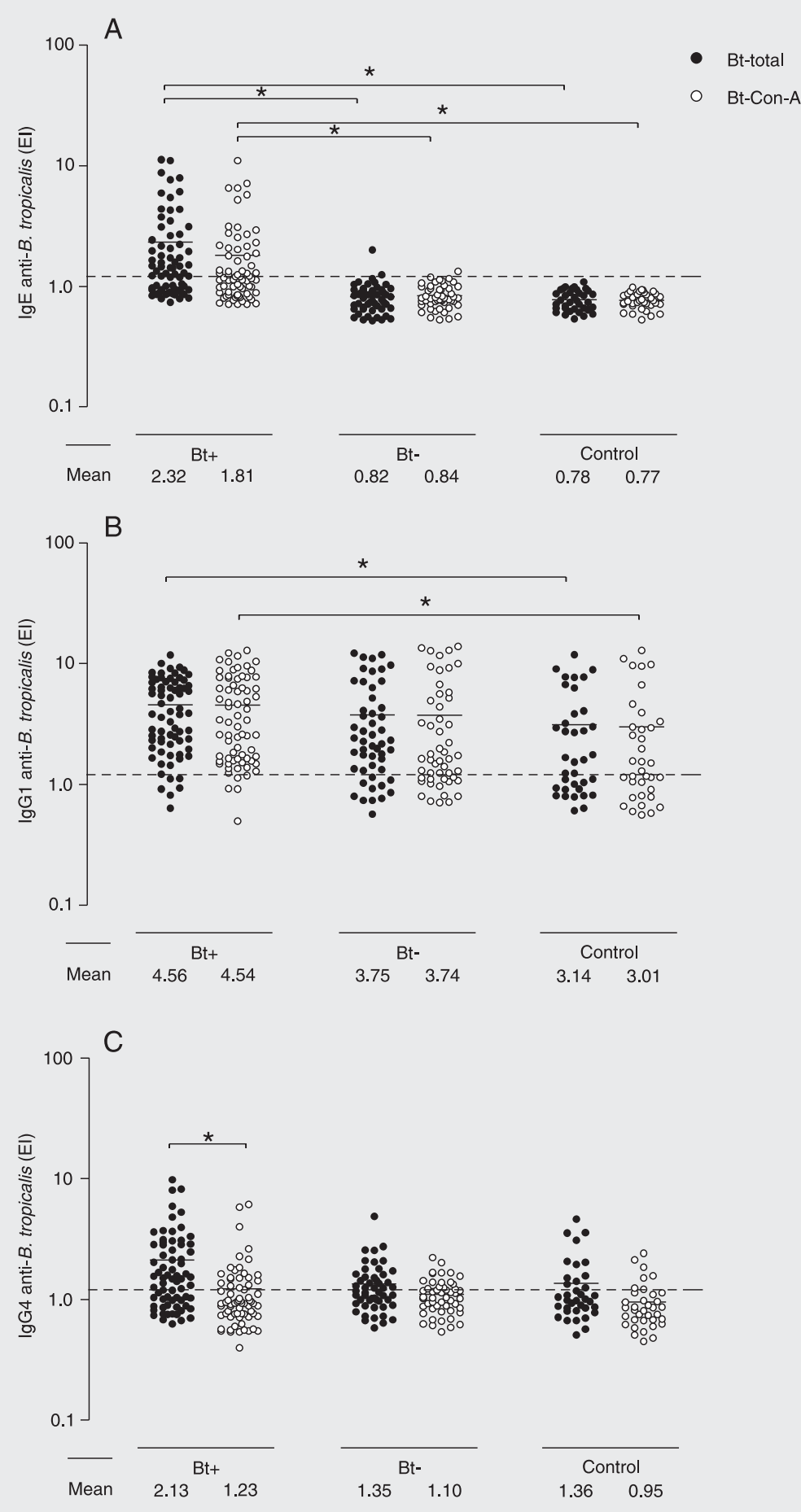

Figure 2. Levels of $\lg \mathrm{E}(\mathrm{A}), \lg \mathrm{G} 1$ (B), and $\lg \mathrm{G} 4$ (C) reactivity to Blomia tropicalis total extract (Bt-total) and concanavalin $A(C o n-A)$ bound fraction (Bt-Con-A). The antibodies were determined in serum samples from patients with a positive $(\mathrm{Bt}+, \mathrm{N}=70)$ and negative (Bt-, $N$ $=51)$ skin prick test in response to $B$. tropicalis and also from control subjects $(N=36)$. Data are reported as ELISA index (EI). Each horizontal bar indicates the mean value obtained for each group. The dashed horizontal line indicates the cut-off of the assay $(E I=1.2)$. ${ }^{*} P<0.05$ for the comparisons (Kruskal-Wallis test using the Dunn's multiple comparison test). (group $\mathrm{Bt}+$ ) to $\mathrm{B}$. tropicalis (Figure 4). A significantly high and positive correlation was observed between $\operatorname{IgE}\left(\mathrm{r}_{\mathrm{s}}=0.860 ; \mathrm{P}<\right.$ $0.0001), \operatorname{IgG} 1\left(\mathrm{r}_{\mathrm{s}}=0.883 ; \mathrm{P}<0.0001\right)$ and IgG4 $\left(r_{\mathrm{s}}=0.790 ; \mathrm{P}<0.0001\right)$ antibodies to Bt-total and Bt-Con-A extracts. When analyzing the frequency of concomitantly positive antibodies to both extracts in $\mathrm{Bt}+$ patients, a significantly higher double-positive rate was found for IgG1 (89\%) compared to similar rates for IgE (44\%) and IgG4 (31\%; $\mathrm{P}<0.0001)$. In contrast, the frequency of single-positive antibodies to Bt-total was significantly lower for IgG1 (3\%) compared to $\operatorname{IgE}(16 \% ; \mathrm{P}<0.01)$ and $\operatorname{IgG} 4(30 \% ; \mathrm{P}<$ $0.0001)$, with significant differences also found between $\operatorname{IgE}$ and $\operatorname{IgG} 4(\mathrm{P}<0.05)$. In contrast, the percentage of single-positive antibodies to Bt-Con-A was negligible $(<4 \%)$ for all antibody classes or subclasses.

\section{Specificity of IgE-, IgG1-, and IgG4-ELISA for B. tropicalis}

Specificity of ELISA for IgE, IgG1, and IgG4 antibodies to Bt-total and Bt-Con- $\mathrm{A}$ extracts was demonstrated by competitive inhibition tests using free Bt-total, Bt-Con$\mathrm{A}$, or Dp extracts, and Con-A as irrelevant antigen (Figure 5). All three assays (IgE, IgG1, and IgG4) using Bt-total or Bt-Con-A extract on the solid phase were inhibited in a dose-dependent manner by increasing concentrations of soluble Bt-total and Bt-Con-A extracts, showing parallel inhibition curves even at very low inhibitor concentrations. In addition, cross-reactivity with the Dp extract was observed in all assays for both extracts, notably for IgG1 and IgG4 (Figure $5 \mathrm{~B}, \mathrm{C}, \mathrm{E}, \mathrm{F})$ that reached $>80 \%$ inhibition at $100 \mu \mathrm{g} / \mathrm{mL}$, the highest concentration used. This was similar to homologous inhibition ( 90\%) using Bt-total or Bt-Con-A extracts. However, for IgE antibodies, the heterologous inhibition with the Dp extract ( 70\%) was significantly lower compared to homologous inhibition $(\sim 90 \%$; $\mathrm{P}<0.01)$. Con- 

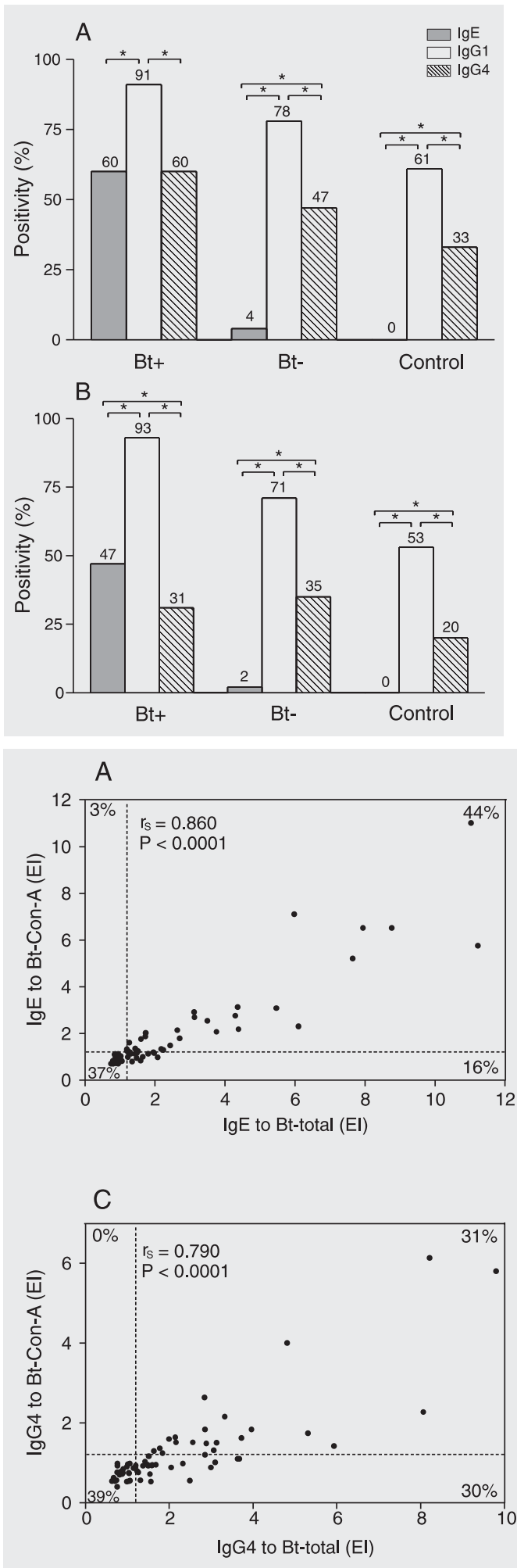

Figure 3. Positivity (\%) of $\lg E$, $\lg G 1$ and $\lg G 4$ antibodies to Blomia tropicalis total extract $(A)$ and to concanavalin $A$ bound fraction (B). The antibodies were determined in serum samples from patients with a positive $(\mathrm{Bt}+, \mathrm{N}=70)$ and negative (Bt-, $\mathrm{N}=51)$ skin prick test to $B$. tropicalis and from control subjects $(\mathrm{N}=36)$. ${ }^{*} \mathrm{P}<0.05$ for the comparisons (Fisher exact test).

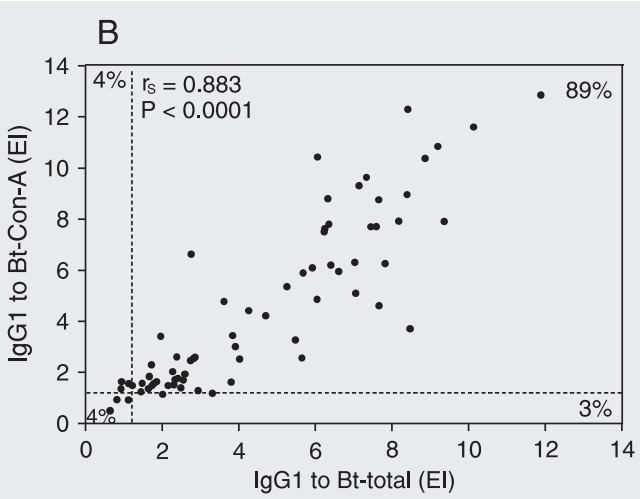

Figure 4. Correlation and association between the levels of $\lg E(A), \lg G 1(B)$, and $\lg G 4(C)$ to Blomia tropicalis total extract (Bt-total) or to concanavalin $\mathrm{A}$ (Con-A) bound fraction (Bt-Con-A), measured by ELISA. The antibodies were determined in serum samples from patients with positive SPT $(\mathrm{Bt}+, \mathrm{N}=70)$ in response to $B$. tropicalis. The correlation coefficient $\left(r_{s}\right)$ was calculated by the Spearman correlation test. The dashed lines indicate the cut-off values (ELISA index, $\mathrm{EI}=1.2$ ) for positivity in each assay. The percentages of doublepositive, double-negative or single-positive antibodies for each extract are indicated in the corresponding corners. For example, in panel A $3 \%$ indicates the percentage of patients with single-positive IgE to BtCon-A extract; $44 \%$ indicates the percentage of patients with double-positive IgE to Bt-total and Bt-Con-A extracts; $16 \%$ indicates the percentage of patients with single-positive IgE to Bt-total extract, and $37 \%$ indicates the percentage of patients with double-negative IgE to Bt-total and Bt-Con-A extracts. 
A that was used as irrelevant antigen did not show any inhibition.

\section{Discussion}

The majority (58\%) of AR patients were sensitized to B. tropicalis, with $52 \%$ presenting positive SPT to both mites (B. tropicalis and $\mathrm{Dp}$ ) and $6 \%$ to $B$. tropicalis only. These results were similar to those of our previous studies carried out in another group of AR patients from the same region (8), confirming that the high rate of this concurrent sensitization has certainly complicated the evaluation of the role of $B$. tropicalis in these patients, particularly in tropical and subtropical regions where both mites coexist.

Glycosylated antigens have been shown to play a crucial role in different pathologies, particularly in fungal infections (11-14) and allergic diseases (15). In this context, we fractionated the Bt-total extract by Con-A Sepharose affinity chromatography resulting in Con-A-unbound (Bt-void) and ConA-bound (Bt-Con-A) fractions. Interestingly, a distinct electrophoretic profile was observed for these two fractions, with Bt-void presenting predominantly low-molecular weight $(<30 \mathrm{kDa})$ components and Bt-ConA presenting predominantly high-molecular weight (>27 kDa) components. The specific
Figure 5. Specificity of ELISA for $\lg E(A, D), \lg G 1(B, E)$ and IgG4 (C, F) antibodies to Blomia tropicalis extracts using competitive inhibition assays. Bt-total, Bt-Con-A, or Dp extracts, or Con-A (irrelevant antigen) were used as inhibitors, serially diluted and incubated with six pooled sera from patients with a positive skin prick test in response to $B$. tropicalis. The antigen-serum mixtures were assayed by ELISA using Bt-total $(A, B, C)$ or Bt-Con-A (D, E, F) extracts on the solid phase. Percent inhibition was calculated as stated in Methods. Bt-total $=B$. tropicalis total extract; Bt-Con-A = concanavalin $\mathrm{A}$ bound fraction from B. tropicalis; $\mathrm{Dp}=$ Derma tophagoides pteronyssinus extract.
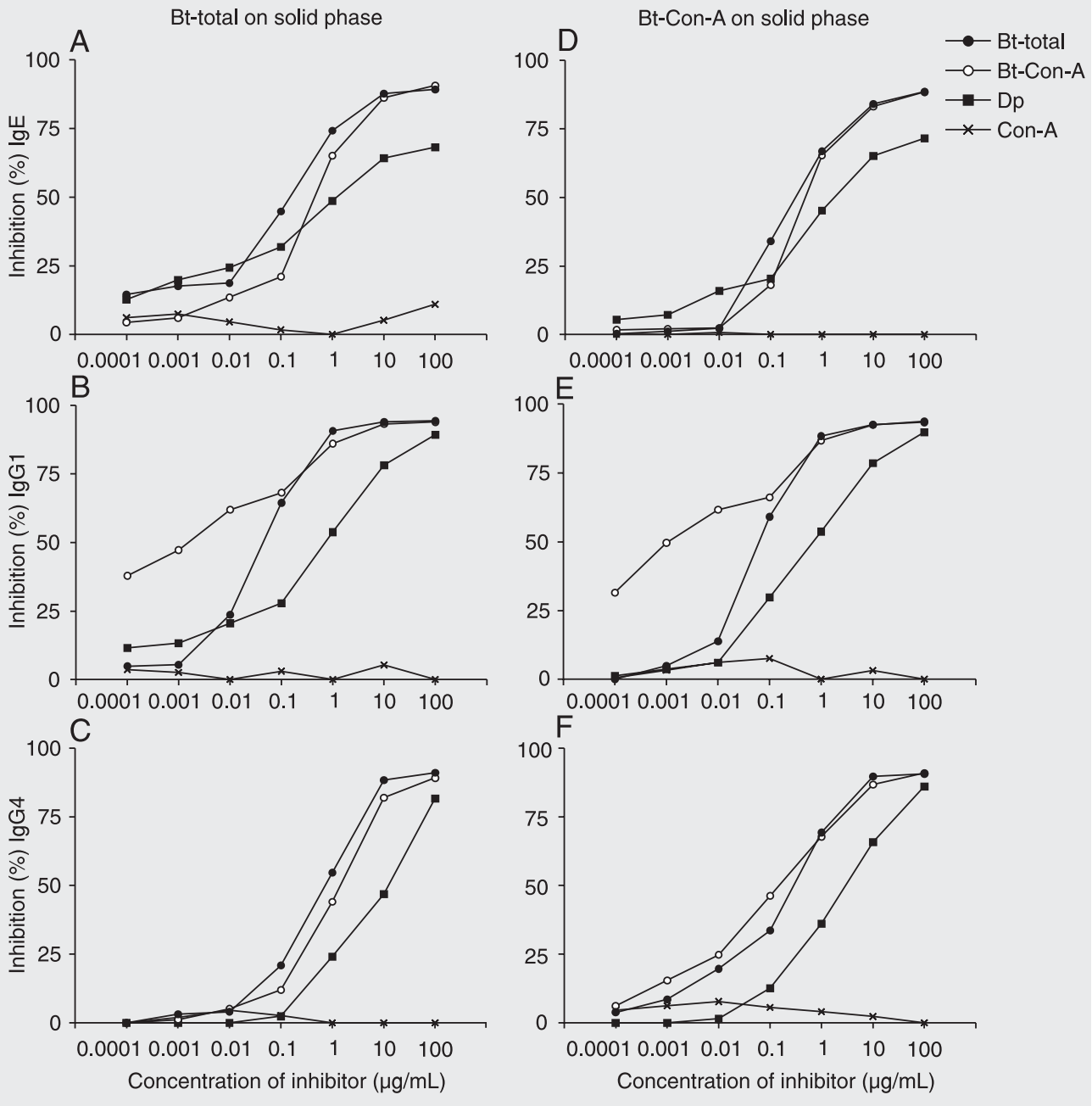
reactivity of these components to $\operatorname{IgE}$ or $\operatorname{IgG}$ subclasses in atopic patients is being investigated by immunoblotting.

In the present study, we analyzed the $\mathrm{IgE}, \mathrm{IgG} 1$, and $\mathrm{IgG} 4$ antibody responses to different $B$. tropicalis antigenic components present in the whole extract (Bt-total) and in the Con-A-binding fraction (Bt-Con-A) in sera from AR patients.

ELISA results showed a similar profile for $\mathrm{IgE}, \mathrm{IgG} 1$ and $\mathrm{IgG} 4$ antibodies directed to both extracts (Bt-total and Bt-Con-A) in different groups of patients, except for Bt+ patients who showed a lower IgG4 reactivity to the Bt-Con-A extract. This fact suggests that different epitopes could be recognized by different antibody classes, with $\operatorname{IgE}$ and IgG1 antibodies recognizing more glycosylated epitopes than IgG4 antibodies. In addition, the significantly higher IgE levels in $\mathrm{Bt}+$ patients as compared to Bt- and control subjects indicate that IgE-ELISA might be considered as an additional method for evaluating the IgE antibody response to $B$. tropicalis in AR patients.

In the present study, the levels of IgG1 and IgG4 antibodies to both extracts were detected in the three groups studied, indicating that these subjects were exposed to repeated antigenic stimuli by $B$. tropicalis allergens. However, levels of specific IgG1 were higher in $\mathrm{Bt}+$ patients than in control individuals, while levels of specific IgG4 showed no significant difference between the groups. These results suggest that specific IgG1 and, to a lesser extent, IgG4 antibodies could be related to natural allergen exposure, thus reflecting a normal consequence of a protective immune response and serving to limit immediate hypersensitivity reactions in allergic patients.

The significant positive correlation and association found between $\operatorname{IgE}, \operatorname{IgG} 1$ and IgG4 antibodies to Bt-total and Bt-Con-A extracts indicate that glycosylated antigens constitute major allergens and may be involved in the allergen sensitization, since
$44 \%$ of $\mathrm{Bt}+$ patients had $\operatorname{IgE}$ antibodies to both extracts, as well as in the maintenance of homeostasis demonstrated by the high proportion $(89 \%)$ of $\mathrm{Bt}+$ patients with $\mathrm{IgG} 1$ antibodies to both extracts.

When analyzing the competitive inhibition studies, ELISAs for IgE, IgG1 and IgG4 antibodies to both extracts (Bt-total and BtCon-A) were shown to be specific tests as demonstrated by high homologous inhibition. However, a cross-reactivity was found with the Dp extract, particularly at high concentrations. This cross-reactivity was partial for IgE and total for IgG1 and IgG4 antibodies to both Bt-total and Bt-Con-A extracts on solid phase, indicating that IgG subclasses rather than IgE are preferentially involved in this cross-reactivity and might be recognizing different epitopes in the allergen extracts.

Arruda et al. (4) have demonstrated a $33 \%$ cross-reactivity of $B$. tropicalis with Dp and a $43 \%$ sequence homology between the Blo 5 and Der $\mathrm{p} 5$ allergens, suggesting that several IgE-binding proteins of B. tropicalis could be partially inhibited by Dp. Additionally, Tsai et al. (9) showed that there was $\operatorname{IgE}$ cross-reactivity between $B$. tropicalis and $\mathrm{Dp}$ as demonstrated by the immunoblotting inhibition results. However, two major B. tropicalis components (14.3 and $27.3 \mathrm{kDa}$ ) could not be inhibited by $\mathrm{Dp}$, indicating that several important $B$. tropicalis allergens need to be better investigated.

Most of the cross-reactivity between $B$. tropicalis and Dp extracts has been shown to be due to the low-molecular weight allergen components, in particular to the BtM/Blo t 5 and Der p 5 (14 kDa) or Blot 3 and Der p 3 (25 $\mathrm{kDa})$ allergens $(20,21)$. In the present study, we demonstrated that there was an important IgE cross-reactivity with the Dp extract even when using the Bt-Con-A extract, suggesting that high-molecular weight allergens could also be involved in this cross-reactivity. Accordingly, in our previous studies (8), AR patients appeared to be preferentially sensi- 
tized to B. tropicalis high-molecular weight allergenic components as compared to lowmolecular weight allergens. Immunoblotting inhibition studies should be performed using the Bt-total and Bt-Con-A extracts to better characterize the components that are crossreactive and non-cross-reactive with Dp.

On the basis of these results, we conclude that Con-A-binding components isolated from B. tropicalis constitute major allergens and are involved in both allergen sensitization (IgE response) and the maintenance of homeostasis (IgG1 and IgG4 responses). In addition, different epitopes may be differentially recognized by antibody classes, with IgE and IgG1 recognizing more glycosylated epitopes than IgG4 antibodies. Finally, IgG1 rather than IgG4 antibodies could be related to natural allergen exposure, reflecting a normal consequence of a protective immune response.

\section{Acknowledgments}

We thank Dr. Federico MontealegreGolcher, Ponce School of Medicine, Ponce, PR, USA, for providing dried samples of Blomia tropicalis and Dermatophagoides pteronyssinus, and Dr. Foued Salmen Espíndola, Priscila Ferreira de Sousa Moreira and Mônica Camargo Sopelete for technical assistance.

\section{References}

1. Arlian LG, Bernstein D, Bernstein IL, Friedman S, Grant A, Lieberman $P$, et al. Prevalence of dust mites in the homes of people with asthma living in eight different geographic areas of the United States. J Allergy Clin Immunol 1992; 90: 292-300.

2. Fernandez-Caldas E, Puerta L, Mercado D, Lockey RF, Caraballo LR. Mite fauna, Der $\mathrm{p} \mathrm{I,} \mathrm{Der} \mathrm{f}$ I and Blomia tropicalis allergen levels in a tropical environment. Clin Exp Allergy 1993; 23: 292-297.

3. Arruda LK, Rizzo MC, Chapman MD, Fernandez-Caldas E, Baggio D, Platts-Mills TA, et al. Exposure and sensitization to dust mite allergens among asthmatic children in São Paulo, Brazil. Clin Exp Allergy 1991; 21: 433-439.

4. Arruda LK, Vailes LD, Platts-Mills TA, Fernandez-Caldas E, Montealegre $\mathrm{F}$, Lin KL, et al. Sensitization to Blomia tropicalis in patients with asthma and identification of allergen Blo t 5. Am J Respir Crit Care Med 1997; 155: 343-350.

5. Mori JC, Pires MC, Galvao CE, Ferreira de MJ, Golcher FM, Montealegre F. Determination of Blomia tropicalis-specific $\operatorname{lgE}$ and $\lg G$ subclasses in atopic dermatitis patients. Allergy 2001; 56: 180-184.

6. Aalberse RC, Van Milligen F, Tan KY, Stapel SO. Allergen-specific IgG4 in atopic disease. Allergy 1993; 48: 559-569.

7. Smith AM, Yamaguchi $H$, Platts-Mills TA, Fu SM. Prevalence of IgG anti-Der $\mathrm{p} 2$ antibodies in children from high and low antigen exposure groups: relationship of $\mathrm{IgG}$ and subclass antibody responses to exposure and allergic symptoms. Clin Immunol Immunopathol 1998; 86: 102-109.

8. Pereira EA, Silva DA, Cunha-Junior JP, Almeida KC, Alves R, Sung $\mathrm{SJ}$, et al. IgE, IgG1, and IgG4 antibody responses to Blomia tropicalis in atopic patients. Allergy 2005; 60: 401-406.

9. Tsai JJ, Wu HH, Shen HD, Hsu EL, Wang SR. Sensitization to Blomia tropicalis among asthmatic patients in Taiwan. Int Arch Allergy Immunol 1998; 115: 144-149.

10. Caraballo L, Puerta L, Martinez B, Moreno L. Identification of allergens from the mite Blomia tropicalis. Clin Exp Allergy 1994; 24 : 1056-1060.

11. Puccia R, Travassos LR, Rodrigues EG, Carmona AK, Oliveira MC, Juliano L. Purification of the specific exocellular antigen gp43 from Paracoccidioides brasiliensis: immunological and proteolytic activi- ties. In: Mareska B, Kobayashi GS (Editors), Molecular biology of pathogenic fungi: a laboratory manual. New York: Telos Press; 1994. p 507-515.

12. Pitzurra L, Vecchiarelli A, Peducci R, Cardinali A, Bistoni F. Identification of a 105 kilodalton Cryptococcus neoformans mannoprotein involved in human cell-mediated immune response. J Med Vet Mycol 1997; 35: 299-303.

13. Gomez BL, Figueroa JI, Hamilton AJ, Diez S, Rojas M, Tobon A, et al. Detection of the 70-kilodalton histoplasma capsulatum antigen in serum of histoplasmosis patients: correlation between antigenemia and therapy during follow-up. J Clin Microbiol 1999; 37: 675-680.

14. Savolainen J. A standardized densitometric immunoblotting analysis of Candida albicans protein allergens. Clin Exp Allergy 1995; 25: 357-363.

15. Baldo BA, Uhlenbruck G. Selective isolation of allergens. I. Reaction of house dust mite extracts with tridacnin and concanavalin A and examination of the allergenicity of the isolated components. Clin Allergy 1977; 7: 429-443.

16. National Heart, Lung, and Blood Institute. International consensus report on diagnosis and management of asthma. Bethesda: Department of Health and Human Services, National Institutes of Health; 1992.

17. Sly RM. New guidelines for diagnosis and management of asthma. Ann Allergy Asthma Immunol 1997; 78: 427-437.

18. Lowry $\mathrm{OH}$, Rosebrough $\mathrm{NJ}$, Farr AL, Randall RJ. Protein measurement with the Folin phenol reagent. J Biol Chem 1951; 193: 265-275.

19. Laemmli UK. Cleavage of structural proteins during the assembly of the head of bacteriophage T4. Nature 1970; 227: 680-685.

20. Caraballo L, Mercado D, Jimenez S, Moreno L, Puerta L, Chua KY. Analysis of the cross-reactivity between BtM and Der $p$, two group 5 recombinant allergens from Blomia tropicalis and Dermatophagoides pteronyssinus. Int Arch Allergy Immunol 1998; 117: 38-45.

21. Yang L, Cheong N, Wang DY, Lee BW, Kuo IC, Huang CH, et al. Generation of monoclonal antibodies against Blo t 3 using DNA immunization with in vivo electroporation. Clin Exp Allergy 2003; 33: 663-668. 